\title{
Finding pulsars with LOFAR
}

\author{
J. van Leeuwen ${ }^{1}$ and B. W. Stappers ${ }^{2}$ \\ 1 Stichting ASTRON, PO Box 2, 7990 AA Dwingeloo, The Netherlands \\ e-mail: leeuwen@astron.nl \\ 2 Jodrell Bank Centre for Astrophysics, School of Physics and Astronomy, University of Manchester, Manchester M13 9PL, UK
}

Received 14 August 2009 / Accepted 23 October 2009

\section{ABSTRACT}

\begin{abstract}
We investigate the number and type of pulsars that will be discovered with the low-frequency radio telescope LOFAR. We consider different search strategies for the Galaxy, for globular clusters and for other galaxies. We show that a 25-day all-sky Galactic survey can find approximately 900 new pulsars, probing the local pulsar population to a deep luminosity limit. For targets of smaller angular size such as globular clusters and galaxies many LOFAR stations can be combined coherently, to make use of the full sensitivity. Searches of nearby northern-sky globular clusters can find new low luminosity millisecond pulsars. Giant pulses from Crab-like extragalactic pulsars can be detected out to over a Mpc.
\end{abstract}

Key words. pulsars: general - telescopes - instrumentation: interferometers

\section{Introduction}

Since the discovery of the first four pulsars with the Cambridge radio telescope (Hewish et al. 1968), an ongoing evolution of telescope systems has doubled the number of known radio pulsars roughly every 4 years: an evolution from a large flat receiver with a fixed beam on the sky (the original Cambridge radio telescope) to focusing dishes (Arecibo - Hulse \& Taylor 1975), often steerable (Green Bank Telescope), on both hemispheres (the Parkes telescope - Manchester et al. 2001), with large bandwidths and multiple simultaneously usable receivers for wider fields of view (Parkes, Arecibo). The types of pulsars discovered have changed accordingly, from slow, bright, single and nearby pulsars (the original four) to fast (young and millisecond pulsars), far-away (globular clusters) or dim pulsars, some of which are in binaries.

The next step in radio telescope evolution will be the use of large numbers of low-cost receivers that are combined interferometrically. These telescopes, the Allen Telescope Array (Bower 2007), LOFAR (Röttgering 2003), MeerKAT (Jonas 2007), ASKAP (Johnston et al. 2008) and the SKA (Kramer et al. 2004), create new possibilities for pulsar research.

In this paper, we investigate the prospects of finding radio pulsars with LOFAR, the LOw Frequency ARray. We outline and compare strategies for targeting normal and millisecond pulsars (MSPs), both in the disk and globular clusters of our Galaxy, and in other galaxies.

\section{LOFAR - the low frequency array}

With the stations operational and the first pulsars detected LOFAR is on track to start operation in 2010. We have evaluated and simulated the LOFAR reference configuration for pulsar searches, and will describe that configuration in some detail below.

Using two different types of dipoles, LOFAR can observe in a low and a high band that range from $30-80 \mathrm{MHz}$ and 110-240 MHz respectively. The sensitivity using the high-band antenna (HBA) is several times that of the low-band antenna (LBA) although their survey speeds are similar due to the larger LBA field of view. The low band is expected to be an exciting new window for exploring radio pulsar behavior (cf. Stappers et al. 2007, for an overview of the possibilities for emission physics and interstellar medium studies), but the impact on a pulsar survey of some of the smearing effects further discussed is so strong in the LBA band, that we will only discuss the HBA half of LOFAR in this paper.

The basic collecting elements are the individual dualpolarization dipoles; each $4 \times 4$ set of these dipoles is combined in an analog beamformer and forms an antenna "tile". Tiles are grouped together in stations; stations farther from the array center are larger than inner stations. In the core, HBA stations are grouped in pairs. The innermost 12 stations are 24 tiles each and are packed tightly in a "superstation". Spread over the 2-km core there are 24 more HBA stations of 24 tiles each (making for 36 core stations in total). These core stations are $32 \mathrm{~m}$ in diameter, but are tapered to an about $30 \mathrm{~m}$ effective diameter to reduce sidelobes. Next there are 18 Dutch "remote" stations that are outside the core and consist of 48 tiles each, while the $\sim 8$ international stations that are spread over Europe use $96 \mathrm{HBA}$ tiles.

At each station the tiles are combined to form up to 8 independently steerable "station beams". With the cumulative data rate out of the station being the limiting factor, the product of the number of beams times their bandwidth cannot exceed $32 \mathrm{MHz}$ (potentially $48 \mathrm{MHz}$ ), e.g.: a station beam set up can range from having a single full-bandwidth station beam to 8 independent beams of $4 \mathrm{MHz}$ each. Station beams are subdivided in $195 \mathrm{kHz}$ channels, and sent to the central processor (CEP) supercomputer for correlation, addition and/or different types of beam forming. As illustrated in Fig. 1 CEP can further combine station beams to form $\sim 128$ 16-bit full-polarisation tied-array beams for the superstation, the core and/or the entire array. That number of formed beams is limited by the maximum CEP output rate, currently 50 Gigabits/s. 


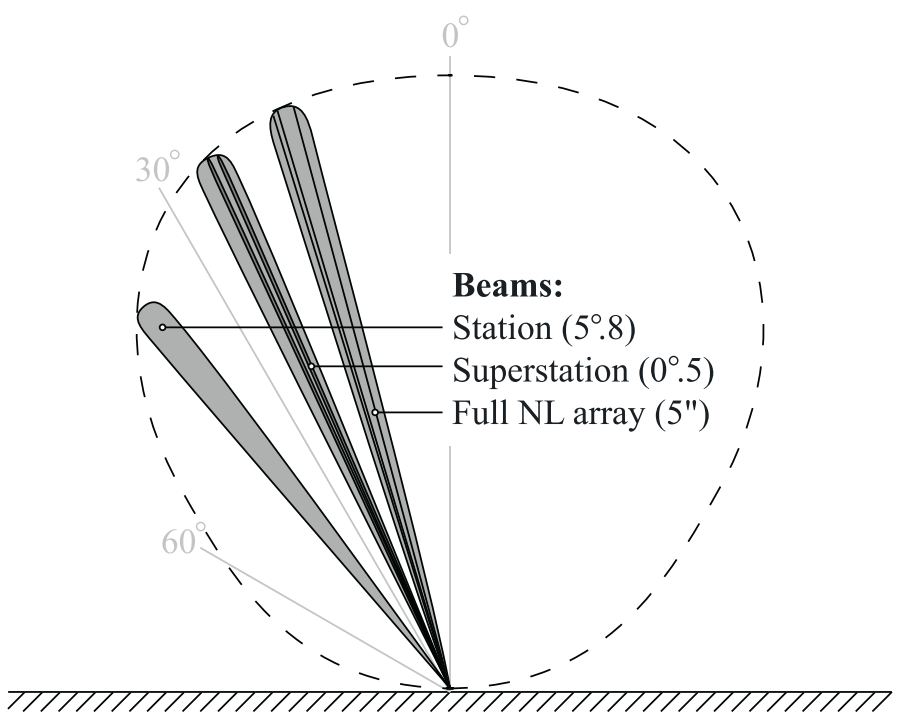

Fig. 1. Illustration of station, superstation and full-array beam patterns at $120 \mathrm{MHz}$. The zenith is at $0^{\circ}$. The dashed envelope is the tile response as shown in Fig. 2. The gains of the different beams are here scaled to be the same. Three stations beams are shown, up to eight can be formed at a given time.

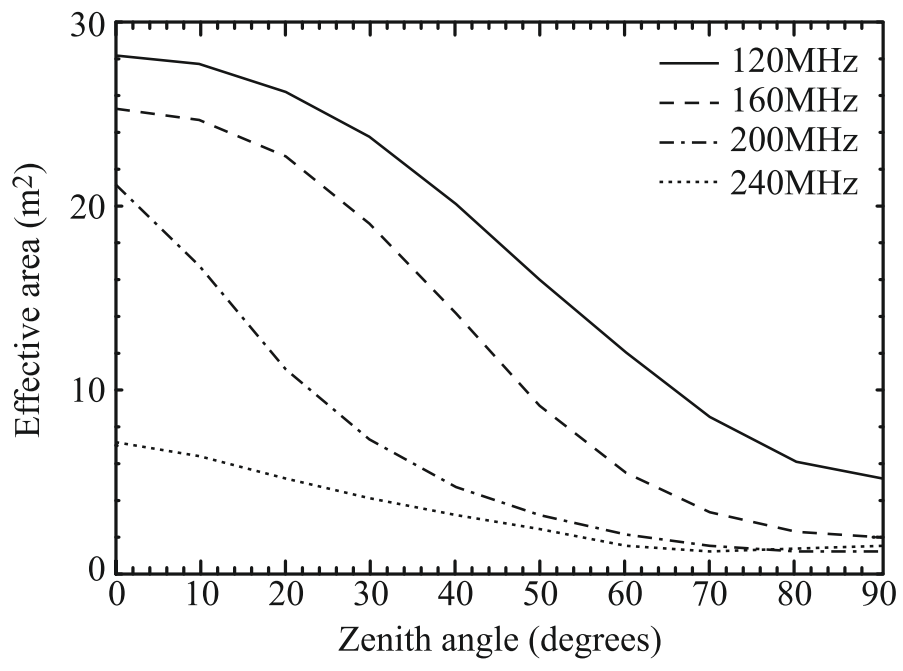

Fig. 2. Simulated effective area per antenna tile versus zenith angle, for 4 different observing frequencies. From Wilhelmsson (2007, private comm.).

The high-band antennas operate in a $110-240 \mathrm{MHz}$ frequency range and are spaced to optimize sensitivity for the low end. They are maximally sensitive toward the zenith. The falloff of effective area $A_{\text {eff }}$ with zenith angle and observing frequency has been well characterized as shown in Fig. 2 (after Wilhelmsson 2007, private comm.) and we will also describe it qualitatively here: at its maximum at $120 \mathrm{MHz}$, the effective area toward zenith is $28 \mathrm{~m}^{2}$ per antenna tile, halving at a zenith angle of $\sim 50^{\circ}$. Toward higher frequencies both quantities decrease until at $240 \mathrm{MHz}$ the maximum effective area is $8 \mathrm{~m}^{2}$ per antenna tile, dropping to half that at $\sim 30^{\circ}$ away from zenith. For the $N_{\text {station }}=36$ stations of the compact core at $120 \mathrm{MHz}$, with their $N_{\text {tiles }}=24$ tiles each, this effective area translates into a theoretical maximum core gain $G_{\max }$ core of

$G_{\text {max }, \text { core }}=\frac{28 m^{2}}{} N_{\text {stations }} N_{\text {tiles }} 2 k \times 10^{-26} \frac{\mathrm{W} \mathrm{s}}{\mathrm{Jy} \mathrm{m}^{2}}=8.8 \mathrm{~K} / \mathrm{Jy}$ where $k$ is Boltzmann's constant in $\mathrm{W} \mathrm{s} \mathrm{m}^{2}$. Finally, the noise temperature $T_{\text {antenna }}$ of the HBA is expected to be $140 \mathrm{~K}$ at $120 \mathrm{MHz}$ and around $180 \mathrm{~K}$ in the upper half of the band (Gunst 2007, private comm.).

\section{Pulsar searches}

\subsection{All-sky surveys}

Although a pulsar survey generally addresses several science questions in parallel, it can be optimized for a specific goal: by using short integration times potential acceleration smearing is kept to a minimum to optimize for finding millisecond pulsars; when aiming to maximise the total number of new pulsars found, a survey should generally focus on the Galactic plane; while for a representative understanding of the local population an all-sky survey that is equally sensitive in all directions is optimal. Here we will focus on such an all-sky survey.

Below we first investigate different beam forming scenarios. These we compare assuming use of a single station beam, at full bandwidth, at the lowest observing frequency of $120 \mathrm{MHz}$. We investigate frequency and band width dependencies in more detail in Sect. 3.1.2.

\subsubsection{Beam forming}

To first order an all-sky pulsar survey is optimized to reach best sensitivity per given overall time. For that one should minimise system noise while maximising collecting area, bandwidth, and integration time per pointing.

For a given survey time and sky area, maximising the time per pointing is equivalent to maximising the instantaneous field of view. This can be done by forming multiple simultaneous beams; while for sparse telescopes like LOFAR, where receivers are spread out over a large area, one can also add stations incoherently instead of coherently. This increases the field of view at the cost of increasing uncorrelated noise over correlated signal (cf. Backer 1999). In the incoherent case the beam on the sky will be larger, but in the coherent case the system is more sensitive.

From the above we can construct the following survey figure of merit FoM for a given observing frequency (see Cordes 2002; Smits et al. 2009, for related definitions):

$F o M=10^{-3}\left(\frac{A}{T_{\text {sys }}}\right)\left(\frac{N_{\text {beams }} \Omega B}{1 \mid N_{\text {stations }}}\right)^{1 / 2}$

where the factor $10^{-3}$ scales the reference $F o M$ to be $1.0 ; A / T_{\text {sys }}$ is the ratio of effective area and system noise equivalent temperature, averaged over the field of view, a ratio indicating telescope gain. $N_{\text {beams }}$ is the number of simultaneous beams formed and $\Omega$ denotes the field of view as derived from the beam size. Here and below we estimate all beam sizes by their full width at half maximum $(F W H M)$ as $1.22 \frac{\lambda}{D} \frac{360}{2 \pi}$ deg with $\lambda$ the observing wavelength, and $D$ the diameter of the element over which the beam is formed (e.g.: a station, the core, or the full array). Then $\Omega=\pi\left(\frac{F W H M}{2}\right)^{2} \mathrm{deg}^{2} . B$ is the observing bandwidth; the righthand denominator is 1 for coherent addition, or $N_{\text {stations }}$ when that number of stations is added incoherently. This FoM is inversely proportional to the minimum detectable flux $S_{\min }$. When comparing between setups, the ratio of survey speeds - the time needed to reach a given sensitivity over the whole sky - is $F o M^{2}$. In Table 1 we list the FoM for three survey set ups discussed below. 
Table 1. Figure of merit FoM per Eq. (2) for the three different survey set ups as described in the text: Full Incoherent, Core Coherent and Superstation Coherent.

\begin{tabular}{lcccccc}
\hline \hline Type & $A / T_{\text {sys }}\left(\mathrm{m}^{2} / \mathrm{K}\right)$ & $N_{\text {beams }}$ & $\Omega\left(\mathrm{deg}^{2}\right)$ & $B(\mathrm{MHz})$ & $1 \mid N_{\text {stations }}$ & $F o M$ \\
\hline Full Incoherent & 260 & 1 & 27 & 32 & 54 & 1.0 \\
Core Coherent & 170 & 128 & 0.006 & 32 & 1 & 0.8 \\
Superstation Coherent & 40 & 128 & 0.23 & 32 & 1 & 1.2 \\
\hline
\end{tabular}

All parameters derived using the lowest observing frequency, $120 \mathrm{MHz}$. In the first column, $A / T_{\text {sys }}$, we use 54,36 and 12 stations respectively, of 24 tiles at $28 \mathrm{~m}^{2}$, and $140 \mathrm{~K}$, while for the Superstation Coherent case we also multiply by 0.70 to correct for the lower sensitivity at the station beam edge.

To outline the various trade-offs involved with these survey setups, we first compare scenarios for coherent versus incoherent addition for the LOFAR core only, and not the total array - given the sparseness of the distribution of remote stations, coherent addition of the entire array would result in an extremely limited field of view.

In the Core Coherent scenario all 36 stations in the $2 \mathrm{~km}$ diameter $D_{\text {core }}$ compact core are combined coherently. In this case $\Omega=0.0060 \mathrm{deg}^{2}$ and the gain is as per Eq. (1). For comparison, each of the individual roughly $D_{\text {station }}=30-\mathrm{m}$ core stations forms a beam on the sky of $27 \mathrm{deg}^{2}$. If added incoherently, the resulting beam is as large as for an individual core station (cf. Fig. 1) but at a factor $\sqrt{N_{\text {stations }}}=6$ decreased sensitivity compared to the coherent case. The factor $\left(\frac{D_{\text {core }}}{D_{\text {station }}}\right)^{2}=\left(\frac{2000 \mathrm{~m}}{30 \mathrm{~m}}\right)^{2}=4.4 \times 10^{3}$ increase in beam area allows for longer integrations by the same factor. For synthesis telescopes that form a single coherent "tied-array" beam, like the Westerbork Synthesis Radio Telescope or the Very Large Array, such a factor would well describe the trade-off; but as the LOFAR CEP can output $N_{\text {beams }}=\sim 128$ coherently added core beams simultaneously versus only a single incoherently added core beam, the factor $F_{t}$ difference in integration times is $F_{t}=\left(\frac{D_{\text {core }}}{D_{\text {station }}}\right)^{2} / N_{\text {beams }}=\left(\frac{2000 \mathrm{~m}}{30 \mathrm{~m}}\right)^{2} / 128=35$. Thus, in this comparison the sensitivity decrease by $\sqrt{N_{\text {stations }}}=\sqrt{36}$ of adding stations incoherently and the sensitivity increase by $\sqrt{F_{t}}=\sqrt{35}$ provided by longer integrations practically cancel out: the FoM for coherent or incoherent addition of just the core is very similar.

Although for the coherent sum the addition of remote stations significantly reduces the field of view, for the incoherent case the entire array can be added without field-of-view penalty. Physically the remote stations are twice the size of the core stations but they can be tapered to the size of the core stations to produce a similar field of view. Adding these 18 remote stations in the above equation produces a FoM for the Full Incoherent scenario of 1.0; that is $\left(F_{t} N_{\text {stations, full }}\right)^{1 / 2} / N_{\text {stations, core }}=(35 \times$ $54)^{1 / 2} / 36=1.2$ times higher than FoM for the Coherent Core case which is 0.8 (Table 1). Yet (only) for the Coherent Core survey producing more simultaneous beams increases the FoM; if $50 \%$ more beams could be formed these two scenarios would be equally sensitive.

Incoherent addition may offer slightly better sensitivity because it allows for long integration times. If however these long integration times are a significant part of a potential pulsar binary orbit (cf. 1-h integrations in our reference incoherent survey, defined below, versus the 2.4-h binary period of PSR J0737-3039, Burgay et al. 2003), the apparent change in pulse period due to the acceleration through the orbit decreases the effectiveness of periodicity searches. At a computational cost different types of acceleration searches can mitigate this decrease in sensitivity (Johnston \& Kulkarni 1991; Ransom et al. 2003). A coherent survey is less prone to this acceleration smearing: to reach the same $S_{\min }$ as the incoherent reference survey with 1-h pointings, the coherent addition survey integration time is down to $3 \mathrm{~min}$. From an efficiency point of view short pointings are also more robust to system errors such as data glitches, and to impulsive radio interference. In contrast to searching a single beam for an incoherent-addition survey, handling $\sim 128$ simultaneous data streams, each at high time resolution and totaling 50 Gigabits/s is a significant computational and data-handling task (Backer 1999; cf. Smits et al. 2009, for the SKA).

In a different coherent survey, Superstation Coherent, 128 tied-array beams from just the superstation (cf. Sect. 2) tile out the complete station beam. Compared to coherently adding the entire core, the superstation beams have larger field of view but are less sensitive. In a grid of 128 of these wider superstation beams many will be close to the less sensitive edges of the station beam, causing for some decrease in sensitivity compared to the narrow Coherent Core beams. For $A / T_{\text {sys }}$ in Table 1 we hence use the average sensitivity out to $F W H M$ for an assumed Gaussian station beam, which is $70 \%$ of the sensitivity for the center of the beam. Yet the relatively large field of view caused by the dense packing of the superstation offsets the limited sensitivity of the lower number of stations now used, producing a FoM of 1.2, compared to 1.0 for the incoherent survey discussed above. Given identical overall survey duration, the integration times for this coherent superstation and the incoherent full array are similar, so there is no reduction in acceleration smearing. Inherent to coherent-addition surveys, the computational task of searching through 128 beams simultaneously and in real-time remains.

Beam forming scenarios have an impact on how accurately sources can be located; this we will address in more detail in Sect. 4 where we discuss follow up.

\subsubsection{Observing frequency}

Determining the optimum observing frequency involves mapping out the trade-off between beam size, source and background brightness, and pulse smearing from dispersion and scattering. Some telescope and pulsar behavior favors low-frequency observing: when moving up from the lowest frequencies the beam size decreases as $v^{-2}$ which limits integration time per pointing in a fixed-time all-sky survey; the effective collecting area falls off with frequency; and the intrinsic pulsar brightness decreases with frequency as $v^{-1.5}$ on average (Malofeev et al. 2000). Other circumstances are more favorable at higher frequencies: the background sky noise decreases steeply as $v^{-2.6}$ (Lawson et al. 1987) and smearing from interstellar dispersion and scattering, which makes the pulsar periodicity harder to detect, is less. Much of the effect of dispersion can be removed by searching over many $\left(\sim 10^{4}\right)$ incoherently dedispersed trial 


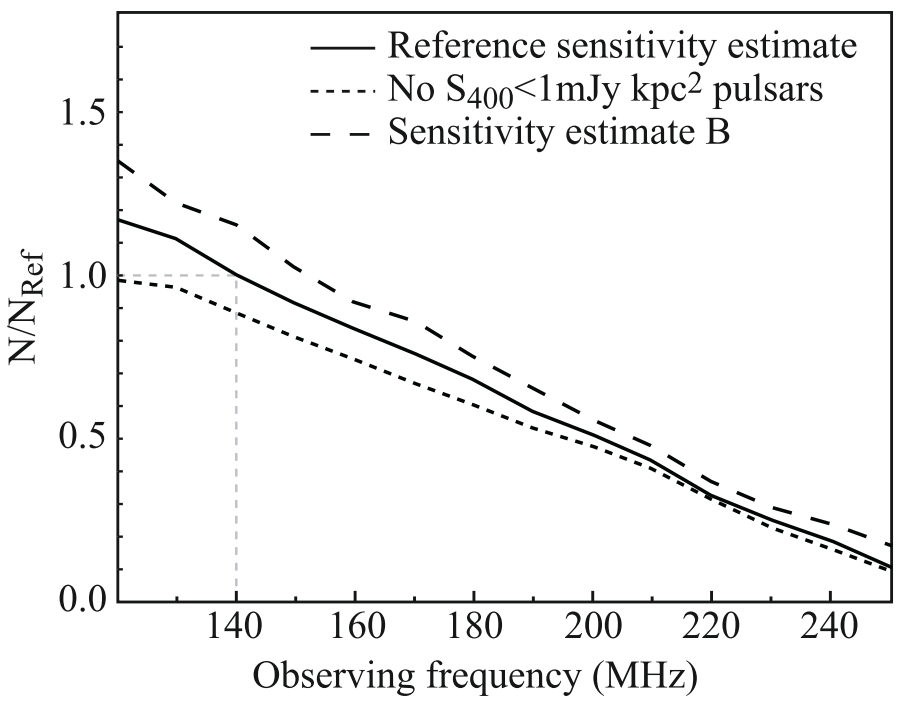

Fig. 3. Number of pulsars detected in simulated surveys at different frequencies and sensitivity estimates, plotted as a ratio over the productivity of our reference 25-day all-sky survey of 1-hr pointings at $140 \mathrm{MHz}$. Towards lower frequencies the yield goes up, mostly caused by the increasing effective area as the wavelength approaches the telescope's optimum at twice the antenna spacing of $1.25 \mathrm{~m}$; equally important is the increasing beam size which allows for longer integrations for the same total survey time.

DMs (Lorimer \& Kramer 2005). No such removal process exists for scatter broadening, where the pulsar emission takes different paths through the interstellar medium with different travel times, resulting in an observed pulse profile that is significantly smeared out. Scattering smearing time increases sharply with decreasing observing frequency (as $v^{-4.5}$, Ramachandran et al. 1997; or alternatively $v^{-3.9}$, Bhat et al. 2004) and with increasing distance to the pulsar (Taylor \& Cordes 1993).

Although the sky background and scatter broadening increase towards lower frequency, we find in our simulations further described below that the total survey productivity, if defined as the total number of pulsars detected (Fig. 3), is mainly determined by the effective collecting area and the beam size. These peak towards the lower edge of the band, leading us to conclude that the survey is most efficient at lowest frequencies. When using a single full-bandwidth $32 \mathrm{MHz}$ station beam this means a central frequency of $140 \mathrm{MHz}$, from here on our reference frequency. As can be seen from Eq. (2), bandwidth can be freely traded for beams with no impact on the FoM. Splitting up the available bandwidth over multiple independently pointing stations beams (keeping beams-bandwidth product equal to $32 \mathrm{MHz}$ as outlined in Sect. 2) and moving each beam to a lower, more sensitive observing frequency may therefore further increase the overall survey output. This does increase integration time per pointing, which may be only beneficial in the Core Coherent scenario where short default integration times could limit sensitivity to intermittent pulsars.

\subsubsection{Sensitivity}

The minimum detectable flux $S_{\text {min }}$ depends on the signal-tonoise ratio at which one accepts a signal as real (SNR), the system temperature $\left(T_{\text {sys }}=T_{\text {rec }}+T_{\text {sky }}\right)$ as it varies over the sky, the number of polarisations $N_{\mathrm{pol}}$, the bandwidth $B$, the integration time $t$, the pulsar period $P$ and pulse width $W$ and the zenithangle dependent gain $G(z)$. Compared to the theoretical gain the real-life gain is always less; although in Eq. (1) we already use the simulated effective area and take aperture efficiency into account there, we apply a conservative factor 0.66 to estimate the real-life gain for our simulations below. Regarding the impact of RFI on the amount of usable bandwidth, testing has shown the radio-interference environment to be relatively clear, and RFI is significantly reduced by the 12-bit digitisation and the many-element interferometer design, especially when elements are combined coherently. As the total bandwidth can furthermore be quickly split up and spaced to avoid channels contaminated by RFI, we expect that the entire specified $32 \mathrm{MHz}$ band (potentially $48 \mathrm{MHz}$ ) will be usable, but below we shall conservatively use $80 \%$ of $32 \mathrm{MHz}$. The potential 1.5 -fold increase in usable bandwidth from 32 to $48 \mathrm{MHz}$ would increase sensitivity by $\sqrt{48 / 32}$. As $S_{\min }$ depends on zenith distance and sky noise, it varies per pointing, but after Dewey et al. (1985) we can estimate a typical value for a 1-min pointing towards the zenith using a coherently formed core beam, for a pulsar with a $10 \%$ duty cycle:

$$
\begin{aligned}
S_{\text {min }} & =S N R\left(\frac{T_{\text {sys }}}{0.66 G_{\max }\left(N_{\mathrm{pol}} 0.8 B t\right)^{\frac{1}{2}}}\right)\left(\frac{W}{P-W}\right)^{\frac{1}{2}} \\
& =10\left(\frac{1.0 \times 10^{3} \mathrm{~K}}{0.66 \times 8.8 \frac{\mathrm{K}}{\mathrm{Jy}}(2 \times 0.8 \times 32 \mathrm{MHz} \times 60 \mathrm{~s})^{\frac{1}{2}}}\right)\left(\frac{1}{3}\right) \\
& =10.3 \mathrm{mJy}
\end{aligned}
$$

For comparison the $S_{\min }$ for incoherently added pointings for our reference survey described below (1-h integrations, 54 stations added) comes to $5.8 \mathrm{mJy}$.

\subsubsection{Simulations}

To determine the number and type of pulsars LOFAR can find, we have used the above characteristics to model the detection of a large number of simulated normal pulsars. For this, we have used a population synthesis code that simulates the birth, evolution, death and possible detection of radio pulsars (for details see Bhattacharya et al. 1992; Hartman et al. 1997; van Leeuwen \& Verbunt 2004). We do not simulate millisecond pulsars, as a realistic treatment of the survey selection effects associated with their binary history would be essential but is beyond the scope of this work. For our simulated pulsars we draw birth velocities from the Lyne \& Lorimer (1994) distribution with its $450 \pm 90 \mathrm{~km} \mathrm{~s}^{-1}$ mean velocity. Initial positions are simulated as in Hartman et al. (1997): heights above the Galactic plane are randomly selected from an exponential distribution with a scale height of $60 \mathrm{pc}$; for the initial distribution in galactocentric radius we use their Eq. (4), modified to scale per unit area:

$p(R) \mathrm{d} R=\frac{R}{R_{\mathrm{W}}} \exp \left(-\frac{R}{R_{\mathrm{W}}}\right) \mathrm{d} R$

where scale length $R_{\mathrm{w}}$ is $5 \mathrm{kpc}$. This modified distribution does not peak as strongly toward the Galactic centre as the original does; for the local population there is no significant change but surveys that sample the inner Galaxy are better reproduced. We next calculate the 3D orbits through the Galaxy. We evolve magnetic fields and periods, determine whether the pulsar is still above the death-line. We estimate the luminosity at $400 \mathrm{MHz}$ (per Hartman et al. 1997; who follow Narayan \& Ostriker 1990). With a spectral index drawn from a Gaussian distribution of width 0.76 around -1.5 (Malofeev et al. 2000, takes luminosity 
Table 2. Name, year, number of detected pulsars $\left(N_{\text {real }}\right)$, number of simulated detected pulsars $\left(N_{\text {sim }}\right)$ and frequency observed at $(v)$ for the eight surveys simulated.

\begin{tabular}{lcccc}
\hline \hline Survey & Year & $N_{\text {real }}$ & $N_{\text {sim }}$ & $v(\mathrm{MHz})$ \\
\hline Jodrell & 1972 & 51 & $20 \pm 2$ & 408 \\
UMass-Arecibo & 1974 & 50 & $39 \pm 4$ & 430 \\
MolongloII & 1978 & 224 & $227 \pm 7$ & 408 \\
UMass-NRAO & 1978 & 50 & $54 \pm 6$ & 400 \\
ParkesII & $1991-1994$ & 298 & $335 \pm 10$ & 400 \\
Cambridge 80MHz & $1993-1994$ & 20 & $27 \pm 5$ & 82 \\
Parkes Multibeam & $1997-2000$ & 987 & $801 \pm 41$ & 1374 \\
LOFAR & $2010-2012$ & - & $1100 \pm 100$ & 140 \\
\hline
\end{tabular}

The error on $N_{\text {sim }}$ is the standard variation in the outcomes when simulating the model described in the text with different random number seeds. The $N_{\text {real }}$ data is taken from Davies et al. (1977); Hulse \& Taylor (1975); Manchester et al. (1978); Damashek et al. (1978); Manchester et al. (1996); Shrauner et al. (1998) and Manchester et al. (2005) respectively. The LOFAR numbers are for the incoherent 25-day reference design described in the text.

turn-overs into account) we scale this luminosity to the observing frequency.

Using the simulated position we look up the sky background temperature (Haslam et al. 1982) and combined with the distance we also estimate the dispersion measure and the scatter broadening from Taylor \& Cordes (1993). Given that both sky background and scatter broadening time increase toward lower observing frequencies we next scale these using power laws with spectral index -2.6 and -4.4 respectively (Lawson et al. 1987; Taylor \& Cordes 1993).

For each of the 8 pulsar surveys in Table 2 we model the sensitivity function versus period, dispersion and scattering smearing, and sky position. We also model the decrease of pulse width and hence detectability with period. By comparing the simulated and real pulsar samples for the first four of the above surveys Hartman et al. (1997) determined the most probable underlying initial model parameters.

We use this best model to determine the yield of a future survey with LOFAR. As tests, we also model the second $81.5-\mathrm{MHz}$ Cambridge survey (Shrauner et al. 1998) to check the validity of our extrapolations to lower frequencies and the Parkes Multibeam pulsar survey (Manchester et al. 2001; Lorimer et al. 2006, hereafter PMB) because of its superior statistics. These tests we describe in some more detail in the two paragraphs below.

For each simulated pulsar, we model if it would be detected by the Cambridge survey given its sky position, dispersion and scatter smearing, and luminosity. We estimate the minimum detectable flux from the $S_{\min }$ versus DM and period plot in Shrauner et al. (1998, their Fig. 3). That figure does not take scattering into account, although for these simulations one realistically should. For each simulated pulsar we therefore determine the "effective" (higher) DM-value that would produce the same smearing as the actual dispersion and scattering do combined. We next determine the sensitivity for the period and that "effective" DM. The sensitivity thus estimated is valid for the survey's median sky background noise of $2 \mathrm{Jy}$, so we next scale it using the actual sky noise for the position of the simulated pulsar. Finally we multiply the sensitivity by the declinationdependent power response (Fig 1a., Shrauner et al. 1998) for the sky position and check if the simulated pulsar is bright enough to be detected. With $27 \pm 5$ simulated pulsars found, our model

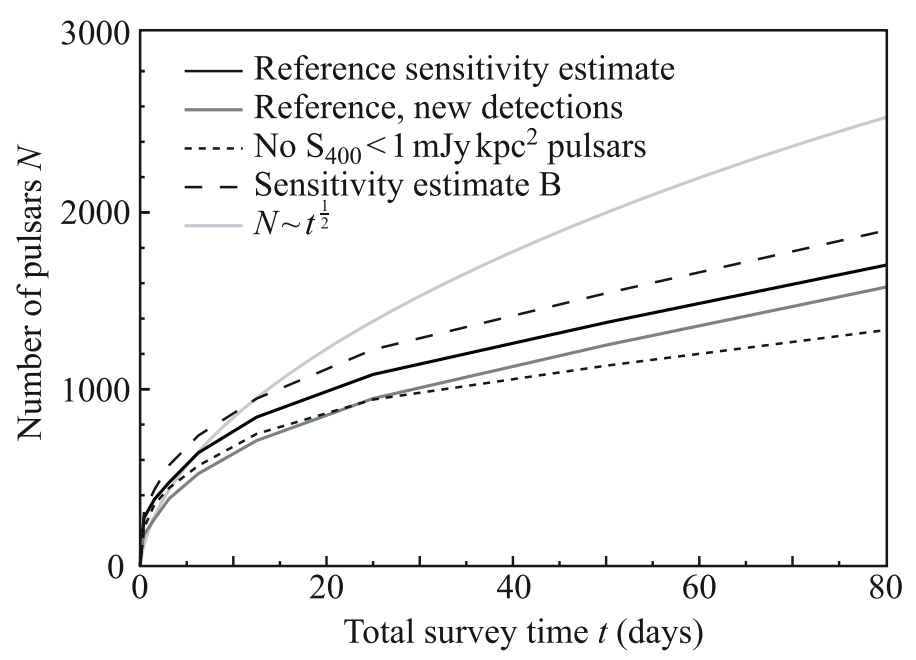

Fig. 4. Simulated number of pulsars detected in an all-sky survey, versus total survey time in days. A survey time of 25 days corresponds to 1-h pointings for incoherently added stations beams.

reproduces the actual tally of 20 pulsars quite well, validating the luminosity, sky-noise and scattering extrapolations to lower frequencies that we also use for the LOFAR predictions.

As a further check on our model, the PMB simulations find $800 \pm 40$ pulsars, which compares reasonably well to the actual number of 987 non-recycled pulsars detected (Manchester et al. 2005, ATNF catalog per Jan. 1, 2009).

For LOFAR, we have evaluated surveys for different telescope parameters, total survey time and minimum sensitivity. Our reference survey is a Full Incoherent survey using 54 stations, 64-min pointings, a gain per Eq. (3) of 0.66 times the theoretical gain, with $80 \%$ of $32 \mathrm{MHz}$ bandwidth at a central frequency of $140 \mathrm{MHz}$. With a single beam such a survey can scan the visible sky in 25 days. In an all-sky survey with this reference set up $1100 \pm 100$ pulsars could be detected (the full curve in Fig. 4), $900 \pm 100$ of which are new. Our sensitivity estimate B uses a less conservative sensitivity of 0.8 times the theoretical gain. In the same overall time it finds about 100-200 more pulsars that are new; i.e. not detected by older surveys we simulate. We do not take into account findings from ongoing surveys such as the GBT350 search project (Hessels et al. 2008; Archibald et al. 2009). Here, and below, the errors quoted for simulation runs indicate variations in output caused by using different initial random seeds. Between simulation runs, the number of new detections is a stable fraction of the total number of detected pulsars, indicating that in the simulations there is a well-defined part of the pulsar population that only LOFAR is sensitive to. In contrast, there are also known pulsars that LOFAR will not be able to detect in this reference survey, mainly due to scattering effects. We have evaluated pointings of 1, 2, 4-256 min duration (see Fig. 4). Here the duration effects the minimum detectable flux; using the figures of merit in Table 1 this incoherent survey can be scaled to the other survey types.

If pulsar luminosity is the limiting factor in the detections, a larger time per pointing $t$ decreases the minimum detectable flux $S_{\text {min }}$ as $t^{-\frac{1}{2}}$, increasing the distance $d$ out to which pulsars can be detected as $t^{\frac{1}{4}}$. At the scale of the Galaxy pulsars are located in a disk; if one could observe pulsars throughout the Galaxy, the number of detectable pulsars is $N \sim d^{2} \sim t^{\frac{2}{4}}$. Locally, pulsars are 

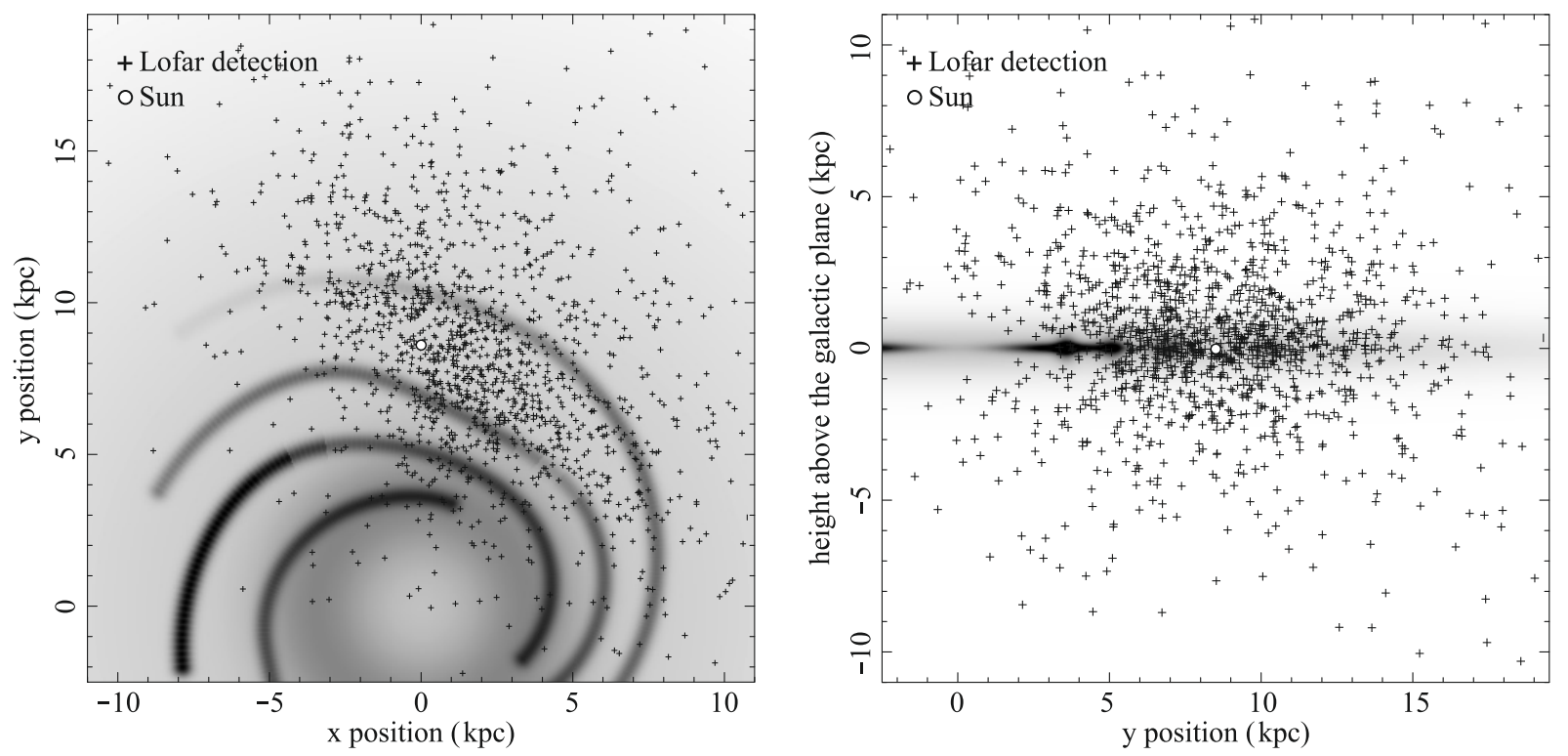

Fig. 5. The 1100 simulated pulsars detected with the reference Galactic LOFAR survey, for 1-h pointings. The scattering interstellar matter (ISM) is shown in gray. Left: projected on the Galactic plane. Right: projected on the plane through the Galactic centre and sun, perpendicular to the disk. The Galactic centre is at $(0,0,0)$. LOFAR probes the local population very well, while the broadening from multi-path scattering on the ISM limits what volume a low-frequency survey like LOFAR probes (ISM modeled after Taylor \& Cordes 1993).

distributed roughly isotropically; then $N \sim d^{3} \sim t^{\frac{3}{4}}$ (cf. Cordes 2002). We find that for LOFAR surveys, $N$ is even less dependent on $t$ than $N \sim t^{\frac{1}{2}}$ because scatter broadening is the limiting factor, not luminosity (cf. Fig. 4, where the $t^{\frac{1}{2}}$ line is scaled to be equal to the reference sensitivity estimate at the 6.25-day point).

Compared to the Parkes Multibeam survey, the pulsars found with LOFAR are different in several ways. The PMB is more strongly focused on the Galactic centre. The range of the population detected by LOFAR is limited by scatter broadening. Because of its higher sensitivity, LOFAR detects more lowluminosity nearby pulsars (Fig. 5a). A lower limit to pulsar luminosity of $1 \mathrm{mJy} \mathrm{kpc}^{2}$ at $400 \mathrm{MHz}$ has been suggested (Lyne et al. 1998, cf. similar lower limit at $1400 \mathrm{MHz}$ in Lorimer et al. 2006); with a spectral index of -1.5 , this compares to a luminosity at $120 \mathrm{MHz}$ of $6 \mathrm{mJy} \mathrm{kpc}^{2}$. If this lower limit exists the reference survey can detect all pulsars that are beamed toward us up to $1 \mathrm{kpc}$. If it does not exist, then this survey will certainly illuminate how many low-luminosity pulsars were formed nearby (cf. PSR J0240+62 in Hessels et al. 2008): a number of importance if one wants to understand the neutron-star birthrate.

Furthermore, LOFAR finds more pulsars out of the Galactic disk (Fig. 5b) as its high sensitivity is unimpeded by scattering in that direction. As pulsars are born in the plane $(z=0)$, this observed $z$-distribution could disclose their much debated birth velocity (cf. Hartman 1997; Arzoumanian et al. 2002).

For the detection of millisecond pulsars (MSPs) scatter broadening will be the limiting factor. Although there is much variation in scattering measure for different lines of sight, on average the scatter broadening at $120 \mathrm{MHz}$ is on the order of $1 \mathrm{~ms}$ for sources with DMs higher than $30 \mathrm{pc} / \mathrm{cm}^{3}$ (using the rough empirical $t_{\mathrm{sc}}=2 \times 10^{9} \mathrm{DM}^{3.8}$ relation between DM and scattering time by Shitov 1994) or distances of about $1 \mathrm{kpc}$ (using distance-scattering relation from Taylor \& Cordes 1993). As many of the currently known Galactic-disk MSPs are within this range of $1 \mathrm{kpc}$ in which scatter broadening does not hinder detection (Manchester et al. 2005), LOFAR could probe the local population to a much lower flux limit.

\subsection{Galactic globular cluster surveys}

In a fixed-time all-sky survey, one can gain field of view at a cost of instantaneous sensitivity by adding the signal of the stations incoherently instead of coherently. When the incoherent FoM is higher (as in Table 1) this trade-off increases the number of detectable pulsars because it allows for more time per pointing. In contrast, specific, smaller regions on the sky with higher densities of radio pulsars can potentially be better targeted with smaller field of view, but significantly higher sensitivity.

Globular clusters fit the description well; they are compact and form regions on the sky with high stellar densities. These high densities also cause globular clusters to contain more binaries and binary products than are found in the disk. This makes globular clusters very good candidates for MSP searches (cf. Lyne et al. 1987). To estimate which clusters are most promising for a LOFAR search, we evaluated several of their properties: location on the sky, dispersion measure (DM), distance $(d)$, and the number of radio pulsars potentially present.

As discussed above for sources in the Galactic plane, scatter broadening is a concern for detecting far-away fast millisecond pulsars. Most globular cluster pulsars have periods of around 2-5 ms (Freire 2008, see Fig. 6). Using the above mentioned DM versus scattering time relations, at $120 \mathrm{MHz}$ such MSPs are detectable up to a DM of about $30-40 \mathrm{pc} / \mathrm{cm}^{3}$. Observing at $200 \mathrm{MHz}$ extends this limit to $60-80 \mathrm{pc} / \mathrm{cm}^{3}$. For the longer period pulsars also present in these clusters, these limits are less of a problem, so we will further investigate globular clusters with DMs up to $100 \mathrm{pc} / \mathrm{cm}^{3}$ below. From low-frequency studies with the Westerbork telescope Stappers et al. (2008) also conclude that the DM versus scattering time relation is uncertain to the extent that some $D M=100 \mathrm{pc} / \mathrm{cm}^{3}$ MSPs may be detectable. We rate the candidate clusters by the expected number of detectable pulsars: assuming $\frac{d \log N}{d \log L}=-1$ (Anderson 1992), this number scales as $d^{-2}$, as the declination-dependent telescope gain $G(z)$ (cf. Fig. 2), and as the collision number $\Gamma \equiv \rho_{\mathrm{c}}^{3 / 2} r_{\mathrm{c}}^{2}$, where $\rho_{\mathrm{c}}$ and $r_{\mathrm{c}}$ are the central density and the core radius, respectively 
Table 3. Name, declination (Dec), distance (d), dispersion measure (DM) observed or ${ }^{+}$modeled after Taylor \& Cordes (1993), core radius $\left(r_{\mathrm{c}}\right)$, collision number $(\Gamma)$ and number of detected pulsars $(N)$ for the ten highest-ranking candidates for a LOFAR globular cluster survey.

\begin{tabular}{lcccccc}
\hline \hline Name & $\begin{array}{c}\text { Dec } \\
\text { deg }\end{array}$ & $\begin{array}{c}d \\
\mathrm{kpc}\end{array}$ & $\begin{array}{c}\mathrm{DM} \\
\mathrm{pc} / \mathrm{cm}^{3}\end{array}$ & $\begin{array}{c}r_{\mathrm{c}} \\
\mathrm{arcmin}\end{array}$ & $\Gamma$ & $N$ \\
\hline M15 & +12 & 10.3 & 67.3 & 0.07 & 665 & 8 \\
M92 & +43 & 8.2 & $29^{+}$ & 0.23 & 106 & \\
M5 & +02 & 7.5 & 30.0 & 0.42 & 79 & 5 \\
M10 & -04 & 4.4 & $43^{+}$ & 0.86 & 28 & \\
M13 & +36 & 7.7 & 30.4 & 0.78 & 39 & 5 \\
M3 & +28 & 10.4 & 26.3 & 0.55 & 66 & 4 \\
M2 & -01 & 11.5 & $29^{+}$ & 0.34 & 120 & \\
M14 & -03 & 9.3 & $76^{+}$ & 0.83 & 54 & \\
Pal 2 & +31 & 27.6 & $97^{+}$ & 0.24 & 209 & \\
M12 & -02 & 4.9 & $39^{+}$ & 0.72 & 10 & \\
\hline
\end{tabular}

Data from Harris (1996) and Freire (2008).

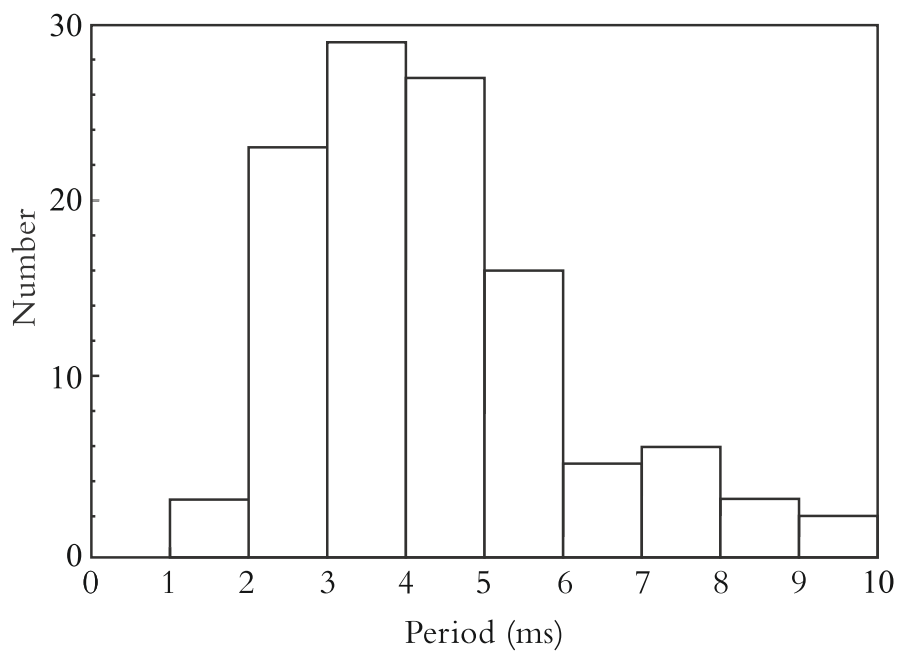

Fig. 6. Periods of the 115 MSPs in globular clusters with periods less than $10 \mathrm{~ms}$. There are another 23 pulsars with longer periods (Freire 2008).

(Verbunt 2003). The sources that are most promising according to this scaling are listed in Table 3. The half-mass radius of each fits within a LOFAR coherent core beam (cf. Fig. 7 for highestranking candidate $M$ 15), which means we can now use the full gain of the coherent addition.

In general, searching globular clusters more deeply than done previously requires integrating for several hours and analyzing the data with multiple binary-acceleration search techniques (see Ransom et al. 2005, and references therein). Assuming an MSP spectral index of -1.7 (Kramer et al. 1998) the weakest pulsar in M15 (B2127+11G, $0.13 \mathrm{mJy}$ at $430 \mathrm{MHz}$, from Anderson 1992) is $1.1 \mathrm{mJy}$ at $140 \mathrm{MHz}$; in a 10-hour pointing with the compact core at $140 \mathrm{MHz}$, the minimum detectable flux (Eq. (3) at M15's zenith angle of $40^{\circ}$ ) is $0.5 \mathrm{mJy}$. Thus far, the luminosities of the pulsars in M15 follow $\frac{\mathrm{d} \log N}{\mathrm{~d} \log L}=-1$. If this relation extends to lower luminosities, observing up to a minimum flux of $0.5 \mathrm{mJy}$ could yield several new MSPs.

\subsection{Pulsars in other galaxies}

The next over-dense regions on the sky are galaxies. Their distance is a problem, but compared to globular clusters, galaxies have several advantages for a LOFAR pulsar survey. They are

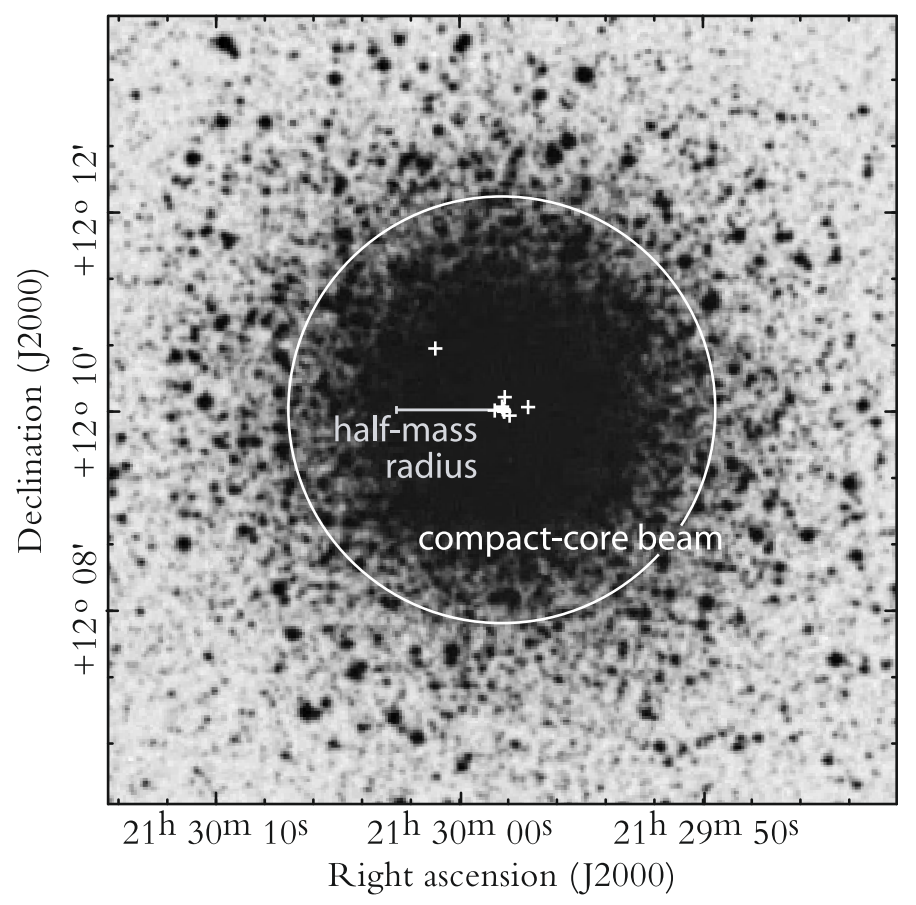

Fig. 7. Beam setup for a MSP survey of M15, the globular cluster with the highest success probability in Table 3 . The cluster half-mass radius falls well within the core beam. The crosses mark the 8 pulsars currently known (Anderson 1992).

distributed equally over both hemispheres, while most globular clusters are invisible from the central telescope site in The Netherlands; and if visible face-on and located in the part of the sky that is pointed away from our Galactic disk, the scatter broadening is relatively low.

In the relatively nearby galaxy $\mathrm{M} 31$, a periodicity search based on a 10 hour pointing with the compact core at $140 \mathrm{MHz}$ could detect all pulsars more luminous than $\sim 200 \mathrm{Jy} \mathrm{kpc}^{2}$ (see Eq. (3)), which is comparable to the top end of the luminosity distribution in our own galaxy. Any M 31 pulsars that emit giant pulses (Argyle \& Gower 1972) could be discovered more easily through these giant pulses than through their periodicity if the flux ratio between giant and normal pulses exceeds $10^{5}$ (McLaughlin \& Cordes 2003). Two young pulsars, the Crab pulsar B0531+21 and LMC pulsar B0540-69 (Staelin \& Reifenstein 1968; Johnston \& Romani 2003), are known to emit such giant pulses and the former was indeed discovered through them. Compared to the regular periodic emission, giant pulses show a steeper spectral index ( -3.0 to -4.5 , Voûte 2001; McLaughlin \& Cordes 2003) making giant-pulse searches especially attractive for a low-frequency telescope like LOFAR. Using the McLaughlin \& Cordes (2003) maximum distance estimator for giant-pulse detection in 1-h pointings

$d=0.85 \mathrm{Mpc}\left(\frac{5 \mathrm{Jy}}{S_{\mathrm{sys}}}\right)\left(\frac{S_{\mathrm{GP}}}{10^{5} \mathrm{Jy}}\right)^{1 / 2}\left(\frac{B}{10 \mathrm{MHz}}\right)^{1 / 4}$

with $S_{\text {sys }}=G_{\text {core }} / T_{\text {sys }}=(0.8 \times 8.8 \mathrm{~K} / \mathrm{Jy}) /\left(1.0 \times 10^{3} \mathrm{~K}\right)=140 \mathrm{Jy}$, bandwidth $B=32 \mathrm{MHz}$ and scaling the giant pulse flux with spectral index -3.0 , the Crab pulsar would be detectable out to $\sim 1.5 \mathrm{Mpc}$.

Detection of a handful of pulsars in each of several nearby galaxies would enable comparison of the top end of their luminosity functions, and potentially show a relation with galaxy 
Table 4. Name, distance $(d)$, size on the sky $(s)$, inclination angle $(i)$, Galactic latitude $(g b)$ and $\operatorname{logarithm}$ of the mass $(\log M)$ for the ten highest-ranking candidates for a LOFAR extragalactic pulsar survey.

\begin{tabular}{lccccc}
\hline \hline Name & $\begin{array}{c}d \\
\text { Mpc }\end{array}$ & $\begin{array}{c}s \\
\operatorname{arcmin}\end{array}$ & $\begin{array}{c}i \\
\text { deg }\end{array}$ & $\begin{array}{c}g b \\
\operatorname{deg}\end{array}$ & $\begin{array}{c}\log M \\
M_{\odot}\end{array}$ \\
\hline M 31 & 0.7 & 193 & 78 & -22 & 11.4 \\
M 81 & 1.4 & 22 & 60 & 41 & 10.7 \\
M 33 & 0.7 & 56 & 56 & -31 & 10.1 \\
M 94 & 4.3 & 12 & 33 & 76 & 10.8 \\
NGC 2403 & 4.2 & 23 & 62 & 29 & 10.7 \\
NGC 4236 & 2.2 & 19 & 73 & 47 & 9.8 \\
NGC 4244 & 3.1 & 15 & 90 & 77 & 10.0 \\
NGC 4395 & 3.6 & 13 & 38 & 82 & 10.1 \\
NGC 3077 & 2.1 & 5 & 43 & 42 & 9.1 \\
UGC 7321 & 3.8 & 5 & 90 & 81 & 9.7 \\
\hline
\end{tabular}

Data taken from Tully (1988).

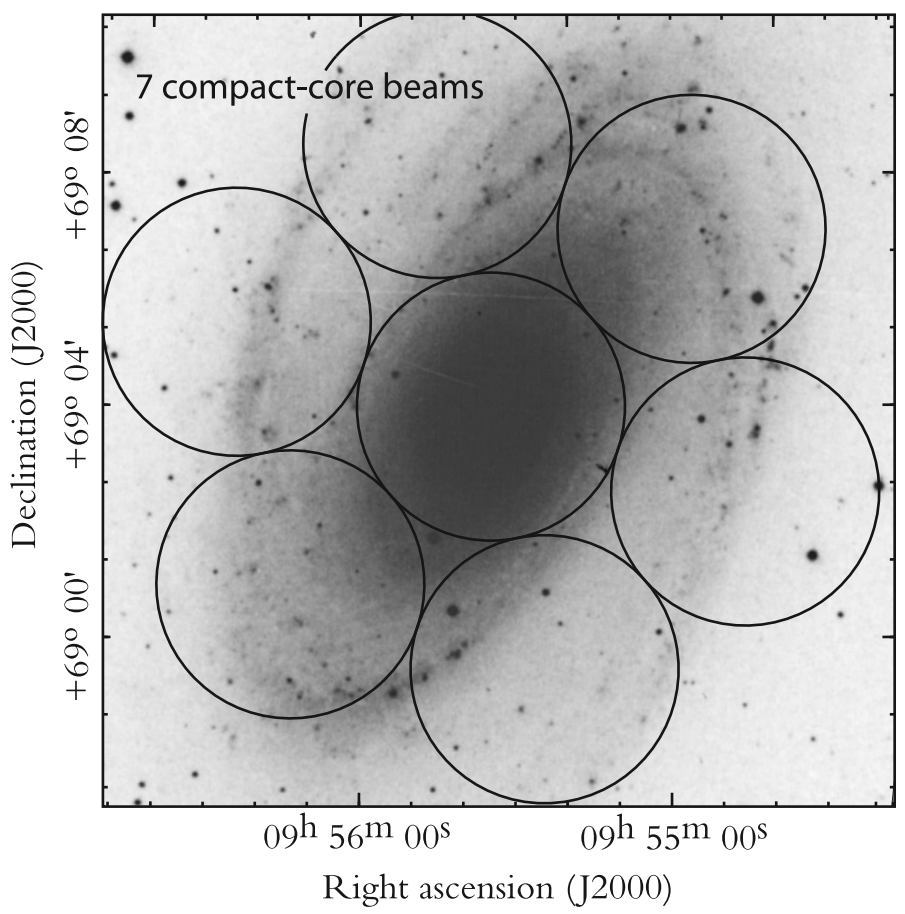

Fig. 8. Seven coherently formed beams from the 2-km LOFAR core projected on candidate galaxy M 81 at their FWHM.

type. Through pulse scattering and dispersion these pulsars will also probe intergalactic matter.

Like for the globular clusters above we have ranked target galaxies by distance, size on the sky and expected number of sources (here assumed to scale linearly with mass). We select galaxies that are within $5 \mathrm{Mpc}$ from the Earth and that are not too strongly scatter-broadened given their Galactic latitude $g b$ and inclination angle $i$ (requiring $|g b|+90-i>30$ ). In Table 4 we list the 10 highest-ranked candidates. For most of these highest-ranked galaxies the core can be captured with a single compact-core beam (cf. Fig 8), or the whole galaxy by a pattern of 7 beams.

\section{Discussion}

\subsection{Steep spectrum sources}

Generally pulsars are increasingly bright toward lower frequencies, but this steep spectrum of pulsars typically flattens out or turns over at a frequency between 100 and $250 \mathrm{MHz}$ (Malofeev et al. 1994). There is, however, a small fraction of pulsars for which no such spectral break is observed down to frequencies as low as $50 \mathrm{MHz}$. Sources with a spectral index steeper than the spectral index of the sky background of -2.6 (e.g. PSR B0943+10, spectral index -4.0; Ramachandran \& Deshpande 1994) will be more easily detectable by a telescope like LOFAR, producing quantitative input for radio emission models.

\subsection{Follow up}

With high sensitivity most important and field of view not a consideration, follow up timing (the determination of pulsar and potential binary parameters, cf. Lorimer \& Kramer 2005) will use the entire core, and potentially more of the LOFAR array if it can be reliably phased up, for beam forming. Given the small $3^{\prime}$ field of view, such follow up needs very accurate candidate source positions. Determining the source position that accurately also directly facilitates timing follow-up by eliminating position as a variable.

In some of the above surveys sources are only localised to within the $30^{\prime}$ superstation or $5^{\circ} .8$ station beams (cf. Sect. 3.1.1). Detections can be more accurately located later by following up with many, smaller tied-array beams. As the DM is now known the total computational burden of searching through these many tied-array beams is not as problematic as for an entirely coherent survey. For example, we find that about $50 \%$ of incoherentsurvey pointings will have sources brighter than the reference $S_{\min }$. Each of these station-beams pointings could subsequently be filled out with 128 coherent superstation beams in about $1 / 3$ rd of the original total survey time $\left(50 \%\left(\frac{F o M_{\text {Full }}}{F o M_{\text {Superstation }}}\right)^{2}=0.36\right.$, cf. Eq. (2) and Table 1) to reach the same $S_{\text {min }}$. This would provide the necessary first conformation observation but also an improved position. In a next step 128 compact-core beams can then similarly tile out the superstation beam in which the source is located to provide full localisation, and the first timing data.

Once sources are properly localised, long-term follow up timing uses tied-array beams. From our simulations we find that in the incoherent reference survey about $80 \%$ of sources have nearest neighbors at less than one $F W H M$ station beam away and hence these pairs of sources can be timed simultaneously. $18 \%$ and $12 \%$ of simulated pulsars are in groups of 3 and 4 per station field-of-view respectively. In total the follow up needs to re-observe about $50 \%$ of the survey pointings to time each newly found pulsar once. Sub-arraying or trading bandwidth for independently steerable station beams could potentially increase overall follow-up timing efficiency. The timing observation should hold at least several hundred pulses for a steady profile to form, which at an average $1 \mathrm{~s}$-pulsar translates to about 10 min integrations. Given the $\sqrt{N_{\text {stations }}}=7.3$-fold increase in gain between incoherent surveying and tied-array followup, a timing run can thus produce high SNR detections for all candidates. 


\subsection{Fast radio transients}

Given the software-telescope nature of LOFAR, piggybacking and simultaneous observing are relatively straightforward to implement. As the "telescope time" concept at LOFAR includes the availability of central signal processing at CEP, observation modes that are not computationally or IO intensive, such as the incoherent station addition mode, are especially suited for parallel observing. This incoherent mode can therefore run commensally with many of the imaging projects (Röttgering et al. 2006; Fender et al. 2007) to continuously scan a large part of the sky for intermittent pulsars such as PSR B1931+24 (Kramer et al. 2006) and millisecond radio transients (Lorimer et al. 2007; Hessels et al. 2009).

\subsection{Survey strategies}

Three types of surveys were presented and their overall efficiency (Table 1) is comparable. When deciding which LOFAR survey to implement, considerations relating to the different integration times, beam sizes and operational requirements in these surveys may thus become more important: Core Coherent has short (order $\sim 1 \mathrm{~min}$ ) integrations, beneficial for finding accelerated systems and for system stability, and provides good localisation. Superstation Coherent and Full Incoherent use of order $\sim 1 \mathrm{~h}$ integrations, and are well suited for finding intermittent pulsars, but with only reasonable and poor initial localisation respectively. Core Coherent and Superstation Coherent produce much higher data rates than Full Incoherent. Tests on the different types of LOFAR data that are currently becoming available may potentially further differentiate between these strategies.

\section{Conclusions}

Because of its large area and field of view, LOFAR can reveal the local population of pulsars to a very deep luminosity limit. A 25-day all-sky survey at $140 \mathrm{MHz}$ would find 900 new pulsars, disclosing the local low-luminosity population and roughly doubling the number of pulsars known in the northern hemisphere. Millisecond pulsars in nearby globular clusters can be detected to lower flux limits than previously possible. Assuming the pulsar population in other galaxies is similar to that in ours, we can detect periodicities or giant pulses from extragalactic pulsars up to several Mpcs away.

\section{References}

Anderson, S. B. 1992, Ph.D. Thesis, California Institute of Technology Archibald, A. M., Stairs, I. H., Ransom, S. M., et al. 2009, Science, 324, 1411 Argyle, E., \& Gower, J. F. R. 1972, ApJ, 175, L89

Arzoumanian, Z., Chernoff, D. F., \& Cordes, J. M. 2002, ApJ, 568, 289

Backer, D. C. 1999, in Perspectives on Radio Astronomy: Science with Large Antenna Arrays, ed. M. P. van Haarlem

Bhat, N. D. R., Cordes, J. M., Camilo, F., Nice, D. J., \& Lorimer, D. R. 2004, ApJ, 605, 759

Bhattacharya, D., Wijers, R. A. M. J., Hartman, J. W., \& Verbunt, F. 1992, A\&A, 254, 198

Bower, G. 2007, in BAAS, 174

Burgay, M., D’Amico, N., Possenti, A., et al. 2003, Nature, 426, 531

Cordes, J. M. 2002,

astro. cornell. edu/ cordes/documents/aosearch.2002.ps.gz

Damashek, M., Taylor, J. H., \& Hulse, R. A. 1978, ApJ, 225, L31

Davies, J. G., Lyne, A. G., \& Seiradakis, J. H. 1977, MNRAS, 179, 635
Dewey, R. J., Taylor, J. H., Weisberg, J. M., \& Stokes, G. H. 1985, ApJ, 294, L25

Fender, R., Wijers, R., Stappers, B., \& LOFAR Transients Key Science Project 2007, in Bursts, Pulses and Flickering: wide-field monitoring of the dynamic radio sky, ed. Tzioumis, Lazio \& Fender, Proceedings of Science, [arXiv:0805.4349]

Freire, P. C. 2008, http://www . naic. edu/ pfreire/GCpsr.html

Harris, W. E. 1996, AJ, 112, 1487, http://www.physics.mcmaster.ca/ resources/globular.html

Hartman, J. W. 1997, A\&A, 322, 127

Hartman, J. W., Bhattacharya, D., Wijers, R., \& Verbunt, F. 1997, A\&A, 322, 477

Haslam, C. G. T., Stoffel, H., Salter, C. J., \& Wilson, W. E. 1982, A\&AS, 47, 1 Hessels, J. W. T., Ransom, S. M., Kaspi, V. M., et al. 2008, AIP Conf. Proc., 983, 613

Hessels, J. W. T., Stappers, B. W., van Leeuwen, J., \& LOFAR Transients Key Science Project 2009, [arXiv:0903.1447]

Hewish, A., Bell, S. J., Pilkington, J. D. H., Scott, P. F., \& Collins, R. A. 1968, Nature, 217, 709

Hulse, R. A., \& Taylor, J. H. 1975, ApJ, 201, L55

Johnston, H. M., \& Kulkarni, S. R. 1991, ApJ, 368, 504

Johnston, S., \& Romani, R. 2003, ApJ, 590, L95

Johnston, S., Taylor, R., Bailes, M., et al. 2008, Exp. Astron., 22, 151

Jonas, J. 2007, in From Planets to Dark Energy: the Modern Radio Universe, October 1-5, The University of Manchester, UK, published online at SISSA, Proceedings of Science, 7

Kramer, M., Xilouris, K. M., Lorimer, D. R., et al. 1998, ApJ, 501, 270

Kramer, M., Backer, D. C., Cordes, J. M., et al. 2004, New Astron. Rev., 48, 993

Kramer, M., Lyne, A. G., O’Brien, J. T., Jordan, C. A., \& Lorimer, D. R. 2006, Science, 312,549

Lawson, K. D., Mayer, C. J., Osborne, J. L., \& Parkinson, M. L. 1987, MNRAS Lorimer, D. R., \& Kramer, M. 2005, Handbook of Pulsar Astronomy (Cambridge University Press)

Lorimer, D. R., Faulkner, A. J., Lyne, A. G., et al. 2006, MNRAS, 372, 777

Lorimer, D. R., Bailes, M., McLaughlin, M. A., Narkevic, D. J., \& Crawford, F. 2007, Science, 318, 777

Lyne, A. G., \& Lorimer, D. R. 1994, Nature, 369, 127

Lyne, A. G., Brinklow, A., Middleditch, J., et al. 1987, Nature, 328, 399

Lyne, A. G., Manchester, R. N., Lorimer, D. R., et al. 1998, MNRAS, 295, 743

Malofeev, V. M., Gil, J. A., Jessner, A., et al. 1994, A\&A, 285, 201

Malofeev, V. M., Malov, O. I., \& Shchegoleva, N. V. 2000, Astron. Rep., 44, 436

Manchester, R. N., Lyne, A. G., Taylor, J. H., et al. 1978, MNRAS, 185, 409

Manchester, R. N., Lyne, A. G., D’Amico, N., et al. 1996, MNRAS, 279, 1235

Manchester, R. N., Lyne, A. G., Camilo, F., et al. 2001, MNRAS, 328, 17

Manchester, R. N., Hobbs, G. B., Teoh, A., \& Hobbs, M. 2005, AJ, 129, 1993

McLaughlin, M. A., \& Cordes, J. M. 2003, ApJ, 596, 982

Narayan, R., \& Ostriker, J. P. 1990, ApJ, 352, 222

Ramachandran, R., \& Deshpande, A. A. 1994, J. Astrophys. Astr., 15, 69

Ramachandran, R., Mitra, D., Deshpande, A. A., McConnell, D. M., \& Ables, J. G. 1997, MNRAS, 290, 260

Ransom, S. M., Cordes, J. M., \& Eikenberry, S. S. 2003, ApJ, 589, 911

Ransom, S. M., Hessels, J. W. T., Stairs, I. H., et al. 2005, Science, 307, 892

Röttgering, H. 2003, New Astron. Rev., 47, 405

Röttgering, H. J. A., R., B., Barthel, P. D., et al. 2006, in Cosmology, Galaxy Formation and Astroparticle Physics on the Pathway to the SKA, ed. H.R. Klöckner, S. Rawlings, M. Jarvis, \& A. Taylor, http://www-astro. physics.ox.ac.uk/LOSKA/CONFERENCE/PROCEEDINGS/

Shitov, Y. P. 1994, BAAS, 26

Shrauner, J. A., Taylor, J. H., \& Woan, G. 1998, ApJ, 509, 785

Smits, R., Kramer, M., Stappers, B., et al. 2009, A\&A, 493, 1161

Staelin, D. H., \& Reifenstein, III, E. C. 1968, Science, 162, 1481

Stappers, B. W., van Leeuwen, A. G. J., Kramer, M., Stinebring, D., \& Hessels, J. 2007, in Proceedings of the 363, WE-Heraeus Seminar on: Neutron Stars and Pulsars, May 14-19, 2006, ed. W. Becker, \& H. H. Huang, MPE Rep., 291,100

Stappers, B. W., Karappusamy, R., \& Hessels, J. W. T. 2008, in 40 Years of Pulsars: Millisecond Pulsars, Magnetars and More, ed. C. Bassa, Z. Wang, A. Cumming, \& V. M. Kaspi, AIP Conf. Ser., 983, 593

Taylor, J. H., \& Cordes, J. M. 1993, ApJ, 411, 674

Tully, R. B. 1988, Nearby galaxies catalog (Cambridge and New York: Cambridge University Press), 221

van Leeuwen, J., \& Verbunt, F. 2004, in Young Neutron Stars and Their Environments, ed. F. Camilo, \& B. M. Gaensler, IAU Symp., 218, 41

Verbunt, F. 2003, in New Horizons in Globular Cluster Astronomy, ed. G. Piotto, G. Meylan, S. G. Djorgovski, \& M. Riello, ASP Conf. Ser., 296, 245

Voûte, J. L. L. 2001, Ph.D. Thesis, University of Amsterdam 